INVESTIGACIÓN

\title{
MEDICIÓN DE LA CONTRIBUCIÓN DE LAS EXPORTACIONES AL PRODUCTO INTERNO BRUTO DE LOS PAÍSES MIEMBROS DE LA COMUNIDAD ANDINA DE NACIONES, MEDIANTE LA ESTIMACIÓN DE ELASTICIDADES E IMPACTOS MARGINALES ${ }^{\star}$
}

\author{
MEASUREMENT OF THE CONTRIBUTION OF EXPORTS \\ TO THE GROSS DOMESTIC PRODUCT OF THE MEMBER \\ COUNTRIES OF THE ANDEAN COMMUNITY OF NATIONS, \\ BY ESTIMATING MARGINAL IMPACTS AND ELASTICITIES
}

\author{
Alexander Carvajal ${ }^{*}$ \\ Oscar Lopez-Camargo**
}

Recibido: 3 de febrero de 2018

Evaluado: 14 de marzo de 2018

Aprobado: 8 de mayo de 2018

* $\quad$ Este texto es producto del Proyecto de investigación "Medición y estimación de la desviación del comercio en los procesos de integración económica en América Latina y el Caribe", financiado por la Universidad Santo Tomás Tunja. El texto corresponde a un artículo científico de resultados parciales del Proyecto de investigación

** Economista, Universidad del Tolima. Especialista en Matemáticas Aplicadas Universidad Sergio Arboleda y Magister en Estadística Aplicada, Universidad de Granada. Actualmente se desempeña como docente en la Universidad Santo Tomás Tunja Colombia. Correos electrónicos: alexander.carvajal@usantoto.edu.co, alcarapel@gmail.com ORCID: 0000-0003-0627-366X

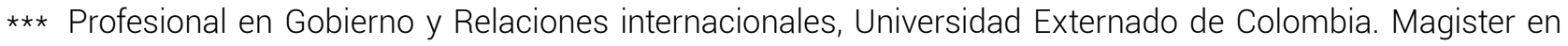
Asuntos Internacionales, Universidad Externado de Colombia. Actualmente se desempeña como docente en la Universidad Santo Tomás Tunja Colombia. Correos electrónicos: oscar.lopezc@usantoto.edu.co,chizacorp@hotmail.com ORCID: 0000-0002-9905-6498 


\section{RESUMEN}

Este documento buscó responder a la pregunta: ¿Cuál es la contribución causal de las Exportaciones (EXP) al Producto Interno Bruto (PIB) en las economías de la Comunidad Andina de Naciones (CAN) ?, por ello, se pretendía medir la contribución de exportaciones al crecimiento del PIB en los países miembros de la CAN; la metodología utilizada fue la estimación de modelos econométricos con las formas funcionales lineal-lineal, lineal-logarítmica, exponencial (logarítmico-lineal), logarítmica-logarítmica y recíproca; En estos modelos, se estableció como variable dependiente el PIB y como variable independiente EXP, luego de estimar los modelos, se calcularon las elasticidades e impactos marginales para cada forma funcional y para cada uno de los países miembros de la CAN (Bolivia, Colombia, Ecuador y Perú). Los resultados obtenidos muestran que existe una inelasticidad EXP del producto PBI y que los impactos marginales son mayores que la unidad en la relación de dependencia de las variables.

Palabras Clave: Elasticidad, Impacto Marginal, Exportaciones, Producto Interno Bruto

\section{ABSTRACT}

This document searched to answer the question: What is the causal contribution of Exports (EXP) to the Gross Domestic Product (GDP) in the economies of the Andean Community of Nations (CAN) ?, therefore, it was intended to measure the contribution of exports to GDP growth in the member countries of the CAN; the methodology used was the estimation of econometric models with the linear-linear, linear-logarithmic, exponential (logarithmic-linear), logarithmic-logarithmic and reciprocal functional forms; In these models, GDP dependent variable and independent variable EXP were established, after estimating the models, marginal elasticities and impacts were calculated for each functional form and for each one of the CAN Member countries (Bolivia, Colombia, Ecuador and Peru). The results obtained show that there is an inelasticity EXP of the GDP product and that the marginal impacts are greater than unity in the relation of dependence of the variables. Keywords: Elasticity, Marginal Impact, Exports, Gross Domestic Product. 


\section{MARCO TEÓRICO}

La teoría económica presenta diferentes posturas que apoyan la hipótesis que argumenta que las exportaciones (EXP) definen el crecimiento económico, reflejado en incrementos del Producto Interno Bruto (PIB). Estas teorías se resumen en dos enfoques teóricos: el primer enfoque denominado Teorías de la Base Económica y enmarcado en las teorías de crecimiento regional; el segundo grupo teórico se conoce como Enfoque de Oferta, el cual se sustenta en indicar que "las exportaciones estimulan el crecimiento económico del país y sus regiones" (Pardo \& Meller, 2002, pág. 2).

Las teorías de la Base Económica esbozan que el crecimiento económico regional es determinado por las actividades de producción de bienes y servicios para exportación (actividades básicas regionales), las formalizaciones más destacadas de esta teoría son la de Homer Hoyt y el modelo Export-Led de Charles Tiebout y Douglas North. Hoyt plantea el crecimiento se mide en términos de empleo $y$, de acuerdo a esto, propone que

$$
\dot{N}_{t}=\dot{N}_{b} \frac{1}{1-b},
$$

Esto es, la tasa de variación del empleo total $\left(\dot{N}_{t}\right)$ es producto de multiplicación de la tasa de variación del empleo en el sector básico $\left(\dot{N}_{\mathrm{b}}\right)$ y el multiplicador del empleo $\frac{1}{1-b}$ (Gaviria, 2010, pág. 12).
El modelo Export-Led reemplaza "las variables físicas del modelo de Hoyt con variables macroeconómicas agregadas -crecimiento, demanda regional, interna y externa- a fin de determinar el crecimiento económico" (Capello, 2016, pág. 123), formalmente se tiene:

$$
Y=\frac{1}{1-(c-m)} \bar{X},
$$

siendo $Y$ nivel de producción, $\bar{X}$ nivel de exportaciones como variable exógena, $\frac{1}{1-(c-m)}$ representa el multiplicador Keynesiano, con $(c-m)$ como la propensión marginal a consumir bienes producidos al interior de la economía y cumpliéndose que $0<c-m<1$. Por tanto, "la renta total (Producción) es un múltiplo de la demanda de exportaciones, el sector básico de la economía" (Gaviria, 2010, pág. 14).

El enfoque de oferta muestra que las economías que impulsan la oferta exportadora tienen mayores niveles de crecimiento que las economías que no realizan este impulso; destaca el trabajo de Bela Balassa (1985) denominado "Las exportaciones, opciones de política y crecimiento económico en países en desarrollo después de la crisis de los combustibles de 1973", en el cual se demuestra que, para el periodo 1973-1979, los países en desarrollo que impulsaron el sector exportador lograron mayores niveles de crecimiento económico (Balassa, 1985, pág. 34). 


\section{METODOLOGÍA}

Bolivia, Colombia, Ecuador y Perú son los países miembros actuales de la Comunidad Andina de Naciones (CAN), la cual ha tenido un crecimiento del PIB Per Cápita (en dólares corrientes) cercanos al $7 \%$ anual, como tasa promedio para el periodo 1970-2010. (Comunidad Andina de Naciones. Secretaría General., 2010, pág. 1) e identificando que uno de los objetivos de la integración económica es el incremento del comercio, es válido plantear la hipótesis que indica que el crecimiento Económico experimentado por la CAN (reflejado en el PIB) puede ser medido de acuerdo al incremento de las EXP.

Para la verificación de esta hipótesis se realizaron los siguientes pasos:

1. Establecimiento de modelos de regresión usados como base para generar mediciones de la relación de causalidad entre EXP y PIB, siendo PIB la variable dependiente y EXP la variable independiente. Los modelos construidos fueron:

a. Modelo lineal-lineal,

b. Modelo lineal-logarítmico,

c. Modelo exponencial (logarítmico-lineal),

d. Modelo de potencia (logarítmico-logarítmico) y

e. Modelo Reciproco.
La utilización de estos modelos se explica porque "las relaciones lineales no son suficientemente generales para todas las aplicaciones económicas" (Wooldridge, 2010, pág. 43). Por lo cual se deben estimar todos los modelos propuestos para lograr conclusionescon resultados robustos.

2. Estimación de las elasticidades e impactos marginales de los modelos obtenidos en el paso previo. La elasticidad se entiende como el cambio porcentual esperado en la variable dependiente $\mathrm{PIB}$, dado que la variable independiente EXP se incrementa 1\%; por otra parte, el impacto marginal indica la variación, en unidades, que experimenta la variable dependiente PIB, ante una variación unitaria de la variable independiente EXP (Pérez \& Fernández, 2009, pág. 75).

Analíticamente, estos modelos, en sus formas funcionales, se expresan así:

a. Modelo lineal-lineal: La ecuación de regresión viene dada por $\widehat{P I B}_{i}=\widehat{\beta}_{0}+\widehat{\beta}_{1} E X P_{i}+\varepsilon_{i}$ la elasticidad se estima mediante: $\eta=\frac{\hat{\beta}_{1} \bar{X}}{\bar{Y}}$ y el impacto marginal obedece a $Y^{\prime}=\hat{\beta}_{1}$.

b. Modelo lineal-logarítmico: La ecuación de regresión viene dada por $\widehat{P I B_{i}}=\widehat{\beta_{0}}+\widehat{\beta_{1}} \log \left(E X P_{i}\right)+\varepsilon_{i}$ la elasticidad se estima mediante: $\eta=\frac{\hat{\beta}_{1}}{\bar{Y}}$ y el impacto marginal obedece $\mathrm{Y}^{\prime}=\frac{\hat{\beta}_{1}}{\bar{x}}$. 
c. Modelo Logarítmico-lineal: La ecuación de regresión viene dada por $\log (\widehat{P I B})_{i}=\log \left(\widehat{\beta_{0}}\right)+\log \left(\widehat{\beta_{1}}\right) \operatorname{EXP}_{i}+\varepsilon_{\text {, }}$ la elasticidad se estima mediante: $\eta=\hat{\beta}_{1} \bar{X}$ y el impacto marginal obedece a $Y^{\prime}=\hat{\beta}_{1} \bar{Y}$.

d. Modelo Logarítmico-logarítmico: La ecuación de regresión viene dada por $\log \left(\widehat{P I B_{i}}\right)=\log \left(\widehat{\beta_{0}}\right)+\widehat{\beta_{1}} \log \left(E X P_{i}\right)+\varepsilon_{i}$, la elasticidad se estima mediante: $\eta=\hat{\beta}_{1}$ y el impacto marginal obedece $\mathrm{a}_{Y^{\prime}}=\frac{\hat{\beta}_{1} \bar{Y}}{\bar{x}}$.

e. Modelo Reciproco: La ecuación de regresión viene dada por $\widehat{P I B_{i}}=\widehat{\beta_{0}}+\widehat{\beta_{1}} \frac{1}{E X P}+\varepsilon_{i}$ la elasticidad se estima mediante: $\eta=\frac{-\hat{\beta}_{1}}{\bar{X} \bar{Y}}$ y el impacto marginal obedece $a_{Y^{\prime}}=\frac{-\hat{\beta}_{1}}{\bar{X}^{2}}$.

f. Donde, $\hat{\beta}_{0}$ y $\hat{\beta}_{1}$ representan el intercepto y la pendiente (respectivamente), en cada modelo estimado; $\varepsilon_{i}$ : indica el residuo del modelo; $\eta$ : representa la elasticidad, $Y$ : señala el impacto marginal y $\bar{X}$ e $\bar{Y}$ indican (respectivamente) el promedio aritmético de los valores de EXP y PIB.

Lo planteado anteriormente, implica que la medición de la relación de causalidad EXP y PIB se mide en términos de elasticidad e impacto marginal. Para todos los residuos de los modelos obtenidos se asume distribución normal y ruido blanco. El periodo de estudio comprende 1990-2016, para todos los países miembros de la CAN. Todos los datos son anuales, y corresponden al PIB real = base 2010 y EXP real =base 2010. Los datos se obtuvieron de la base de datos integrada CEPALSTAT de la Comisión Económica para América Latina y el Caribe. Los datos de las dos variables se encuentran en miIlones de Dólares Americanos.

De acuerdo a lo anterior, el principal objetivo de este escrito se centró en medir la contribución de las exportaciones al crecimiento del PIB en los países miembros de la CAN, y para lograrlo se generaron mediciones básicas entre la relación de las variables de interés mediante la utilización de modelos econométricos y se estimaron medidas de elasticidad e impacto marginal en la relación entre las variables de interés.

\section{RESULTADOS}

Las figuras 1,2, 3 y 4 muestran el comportamiento histórico de las variables PIB real base $=2010$ y EXP reales base $=2010$, para el periodo de estudio (1990-2016), en cada uno de los países miembros de la CAN. Estás figuras son útiles ya que permiten realizar una aproximación visual a la relación de comportamiento de PIB y EXP. Cada grafica se organiza en dos cuadrantes, en el primer cuadrante se observa el PIB real y en el segundo las EXP reales. En el eje horizontal se observan los periodos (años) de estudio y en el eje vertical los valores de PIB o EXP según corresponda. 

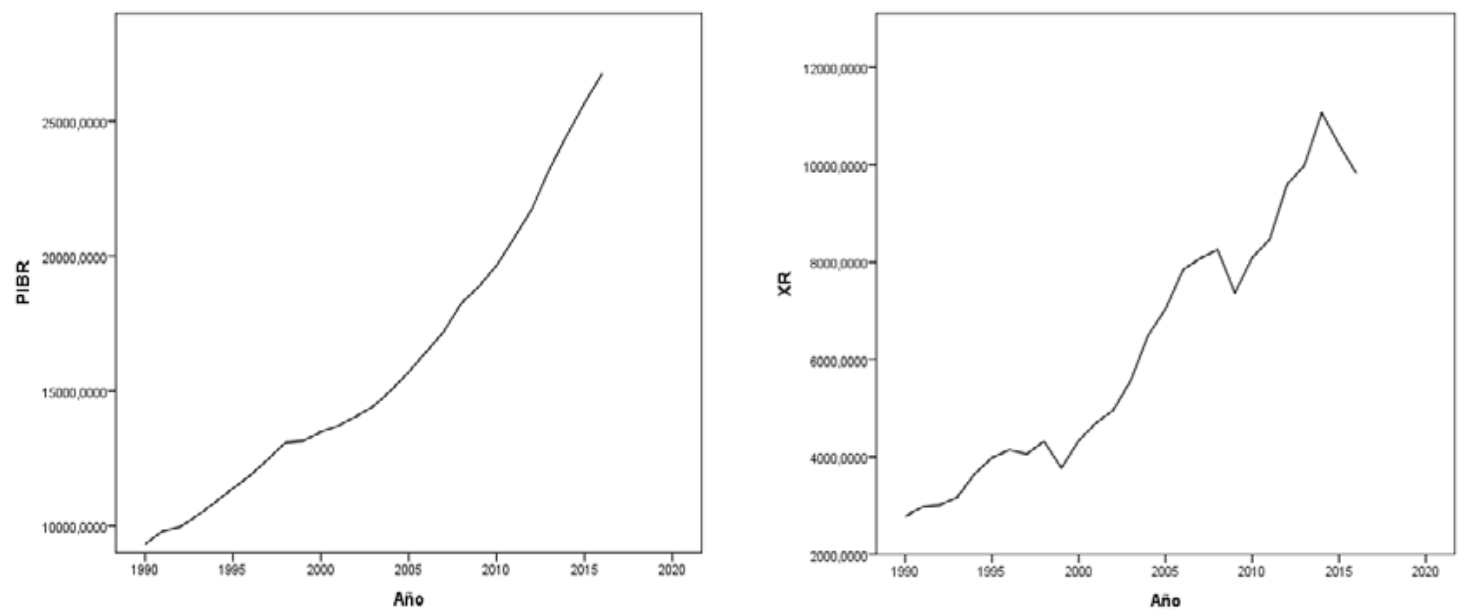

Figura 1. Comportamiento histórico PIB real y Exportaciones Reales. Bolivia.

Fuente: Elaboración propia

En la figura 1 es notorio que, para Bolivia, el PIB real ha tenido un crecimiento sostenido desde finales de la década de los noventa hasta el año 2016, sin embargo, las exportaciones han presentado una tendencia de crecimiento, pero con alguna variabilidad y posterior al año 2014 muestran el inicio de una tendencia decreciente.
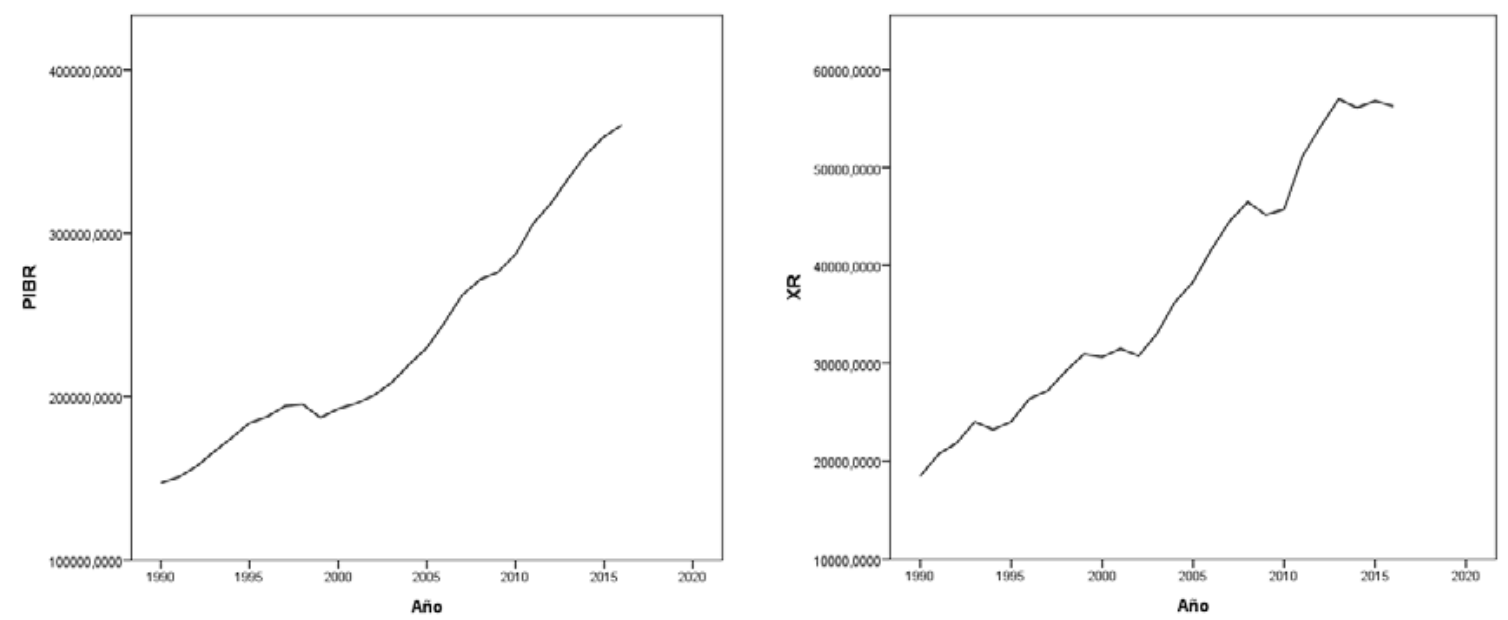

Figura 2. Comportamiento histórico PIB real y Exportaciones Reales. Colombia.

Fuente: Elaboración propia 
En la figura 2 se observa que, en Colombia, el PIB real y las EXP reales presentan una tendencia creciente, pero la variable EXP muestra un comportamiento más irregular que la variable PIB.
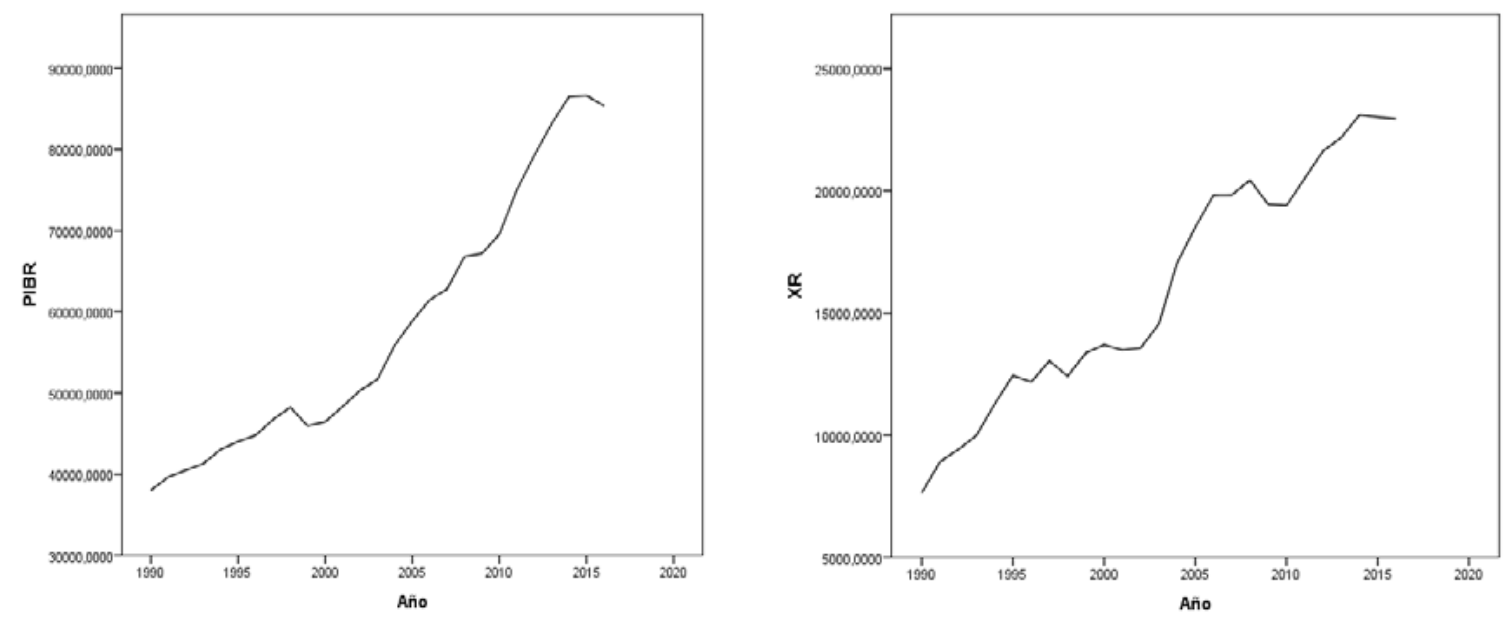

Figura 3. Comportamiento histórico PIB real y Exportaciones Reales. Ecuador.

Fuente: Elaboración propia

La figura 3 hace evidente que, en Ecuador, el PIB real ha crecido de manera uniforme desde inicios de la década de 2.000 hasta el año 2014, año en el cual su valor ha iniciado una tendencia decreciente, por otra parte, las EXP reales han tenido fuertes fluctuaciones durante ese mismo periodo.
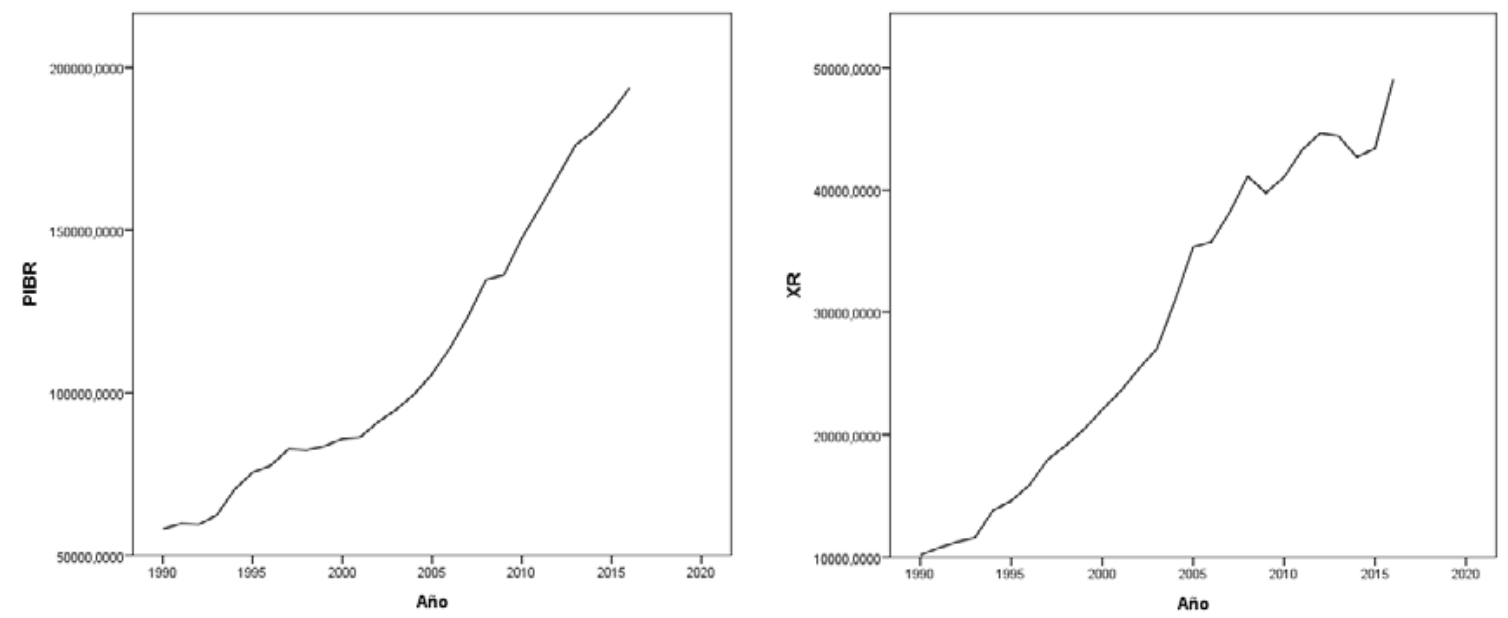

Figura 4. Comportamiento histórico PIB real y Exportaciones Reales. Perú.

Fuente: Elaboración propia 
La figura 4 muestra que, en Perú, el PIB real ha crecido sostenidamente desde inicios de la década del 2.000 hasta 2016 (con una breve interrupción en 2009), las EXP reales crecieron de forma sostenida desde finales de la década del noventa hasta el año 2005, luego ha presentado variabilidad, aunque con tendencia creciente.

En las figuras analizadas es un factor las tendencias crecientes de las variables PIB y EXP, sin embargo, en todos los países las variables EXP presenta mayor variabilidad que la variable PIB. Esta relación es ambigua y no permite establecer con certeza la presencia de relación causal entre las variables.
La tabla 1 muestra los resultados de todos los modelos obtenidos. En la primera columna se indica el país, en la segunda columna se señalan las diversas formas funcionales de los modelos estimados en cada país, en las columnas tres y cuatro se muestran los resultados de los coeficientes estimados (pendiente e intercepto), en la columna 5 se registra el valor del coeficiente de determinación $\left(R^{2}\right)$ de cada uno de las formas funcionales de los modelos estimados, las siguientes columnas muestran los promedios de EXP y PIB para cada uno de los países, finalmente, en las dos últimas columnas se encuentran los valores de la elasticidad e impacto marginal obtenidos.

Tabla 1. Estimaciones diversos modelos.

\begin{tabular}{|c|c|c|c|c|c|c|c|c|}
\hline País & Modelo & Intercepto & Pendiente & $R^{2}$ & $\operatorname{EXP} \bar{X}$ & PIB $\bar{Y}$ & $\eta$ & $Y^{\prime}$ \\
\hline \multirow{5}{*}{ Bolivia } & Lineal & $4,331.59$ & 1.87 & $93 \%$ & 6,221 & 15,987 & 0.73 & 1.87 \\
\hline & Lin-log & - 78,358.60 & $10,913.70$ & $88 \%$ & 6,221 & 15,987 & 0.68 & 1.75 \\
\hline & Log-lin & 8.92 & 0.00 & $94 \%$ & 6,221 & 15,987 & 0.71 & 1.82 \\
\hline & Log-log & 3.71 & 0.68 & $94 \%$ & 6,221 & 15,987 & 0.68 & 1.75 \\
\hline & Reciproco & $26,538.10$ & - 54,636,100 & $80 \%$ & 6,221 & 15,987 & 0.55 & 1.41 \\
\hline \multirow{5}{*}{ Colombia } & Lineal & $37,915.90$ & 5.28 & $96 \%$ & 37,099 & 235,746 & 0.83 & 5.28 \\
\hline & Lin-log & $\begin{array}{l}- \\
1,711,560.00\end{array}$ & $186,096.00$ & $92 \%$ & 37,099 & 235,746 & 0.79 & 5.02 \\
\hline & Log-lin & 11.52 & 0.000022 & $98 \%$ & 37,099 & 235,746 & 0.82 & 5.19 \\
\hline & Log-log & 4.12 & 0.78 & $96 \%$ & 37,099 & 235,746 & 0.78 & 4.96 \\
\hline & Reciproco & $412,540.00$ & $\begin{array}{l}- \\
5,841,980,000\end{array}$ & $83 \%$ & 37,099 & 235,746 & 0.67 & 4.24 \\
\hline
\end{tabular}




\begin{tabular}{|c|l|l|l|l|l|l|l|l|}
\hline País & Modelo & Intercepto & Pendiente & & EXP & PIB & & \\
\hline \multirow{5}{*}{ Ecuador } & Lineal & $7,155.60$ & 3.17 & $92 \%$ & 16,067 & 58,059 & 0.88 & 3.17 \\
\cline { 2 - 9 } & Lin-log & $-381,574.00$ & $45,622.80$ & $85 \%$ & 16,067 & 58,059 & 0.79 & 2.84 \\
\cline { 2 - 9 } & Log-lin & 10.07 & 0.000054 & $96 \%$ & 16,067 & 58,059 & 0.87 & 3.14 \\
\cline { 2 - 9 } & Log-log & 3.34 & 0.79 & $91 \%$ & 16,067 & 58,059 & 0.79 & 2.85 \\
\cline { 2 - 9 } & Reciproco & $98,334.00$ & $-585,119,000$ & $74 \%$ & 16,067 & 58,059 & 0.63 & 2.27 \\
\hline \multirow{5}{*}{ Perú } & Lineal & $20,209.10$ & 3.16 & $90 \%$ & 28,637 & 110,754 & 0.82 & 3.16 \\
\cline { 2 - 9 } & Lin-log & $-648,802.00$ & $74,876.00$ & $81 \%$ & 28,637 & 110,754 & 0.68 & 2.61 \\
\cline { 2 - 9 } & Log-lin & 10.71 & 0.000029 & $96 \%$ & 28,637 & 110,754 & 0.83 & 3.21 \\
\cline { 2 - 9 } & Log-log & 4.40 & 0.70 & $91 \%$ & 28,637 & 110,754 & 0.7 & 2.71 \\
\cline { 2 - 9 } & Reciproco & $175,516.00$ & $1,439,140,000$ & $68 \%$ & 28,637 & 110,754 & 0.45 & 1.75 \\
\hline
\end{tabular}

Fuente: Elaboración propia

En general, todos los modelos estimados, en sus formas funcionales, son significativos (conjunta e individualmente) al $5 \%$ y muestran alto nivel de ajuste. El modelo con menores valores de elasticidad, impacto marginal y $R^{2}$ es el reciproco, en cada uno de los países analizados. El análisis de cada país indica que:

1. En Bolivia, los modelos con mayor $R^{2}$ son el Logarítmico-lineal y el Logarítmico -logarítmico (94\%); el modelo con mayor elasticidad es el Lineal (0.73) y el modelo con mayor impacto marginal es el lineal (1.87).
2. En Colombia, el modelo con mayor $R^{2}$ es el Logarítmico-lineal (98\%); el modelo con mayor elasticidad es el Lineal (0.83) y el modelo con mayor impacto marginal es el lineal (5.28).

3. En Ecuador, el modelo con mayor $R^{2}$ es el Logarítmico-lineal (96\%); el modelo con mayor elasticidad es el Lineal (0.88) y el modelo con mayor impacto marginal es el lineal (3.17)

4. En Perú, el modelo con mayor $R^{2}$ es el Logarítmico-lineal (96\%); el modelo con mayor elasticidad es el Logarítmico-lineal (0.83) y el modelo con mayor impacto marginal es el Logarítmico-lineal (3.21) 


\section{CONCLUSIONES Y DISCUSIÓN}

De acuerdo a los resultados obtenidos se puede afirmar que:

1. Las elasticidades obtenidas muestran, en su mayoría, valores entre 0.5 y 0.8 . Los impactos marginales toman valores en el intervalo 2 y 5 , por lo tanto, se puede argumentar que existe inelasticidad EXP del producto PIB y que los impactos marginales son mayores a la unidad en la relación de dependencia de las variables.

2. El modelo con mejor ajuste (mayor $R^{2}$ ) es el Logarítmico-lineal, en todos los países.

3. El modelo que genera menor inelasticidad es el lineal, esto en Bolivia, Colombia y Ecuador.

4. El modelo que genera mayor impacto marginal es el lineal, esto en Bolivia, Colombia y Ecuador.

Estas conclusiones verifican la validez empírica de las teorías de la base económica (Modelo de Hoyt y Modelo Export-Led) y el enfoque de oferta, sin embargo, se debe tener en cuenta que, al existir inelasticidad exportación del PIB, el crecimiento económico de los países de la CAN no puede ser explicado únicamente por el crecimiento de los valores de EXP. Baltazar (2014) realiza un estudio denominado
"Mercado Interno, exportaciones instituciones y crecimiento económico, un análisis para México" donde llegó a una conclusión similar afirmando que, en México -en el periodo 19962012- "Las exportaciones si bien resultaron estadísticamente significativas, su efecto en el crecimiento es débil" (Baltazar, 2014, pág. 58)

Lo expresado en el párrafo anterior permite plantear que los gobiernos de todos los países de la CAN no deben basar su crecimiento económico supeditados a políticas de fomento exportador, esta afirmación concuerda con lo planteado por Palley, Thomas (2011), quien sugiere que el crecimiento económico debe basarse en un nuevo paradigma fundamentado en el crecimiento de la demanda interna (Palley, Thomas, 2011. Pág.10)

Por otra parte, los resultados obtenidos no deben ser considerados como absolutos, ya que las regresiones planteadas pueden ser espurias (una regresión espuria indica que existe relación matemática entre las variables, pero en la realidad no tienen ninguna relación), por ello, para reafirmar o negar lo aquí obtenido, se propone la construcción de modelos econométricos que utilicen técnicas de cointengración causalidad. La cointegración se define como "la propiedad en la que dos series de tiempo comparten una tendencia estocástica común" (Sant'Anna, Filomena 
\& Caldeira, 2017, Pág.149) y la causalidad se refiere a que una variable es causa de otra variable, en general se pueden aplicar pruebas estadísticas como la causalidad de Wald.

\section{REFERENCIAS}

Balassa, B. (1985). Exports, Policy choices, and economic growth in developing countries after the 1973 oil Shock . Journal of Development Economics , 23-35.

Baltazar, J. (2014). Mercado interno, exportaciones, instituciones y crecimiento económico: Un análisis para México. Tópicos Selectos de Recursos, 49.

Capello, R. (2016). Regional Economics. New York: Routledge.

Comunidad Andina de Naciones. Secretaría General. (2010). Estadísticas de la semana. Evolución del Producto Interno Bruto (PIB) por habitante de la Comunidad Andina de Naciones 1970-2010. Obtenido de http://intranet.comunidadandina. org/Documentos/DEstadisticos/SGde365.pdf
Gaviria, M. A. (2010). Apuntes de Economía Regional. Pereira: Universidad Católica Popular de Risaralda.

Pardo, A., \& Meller, P. (2002). El Rol de las Exportaciones en el Crecimiento Económico Regional. Santiago, Chile: Universidad de Chile.

Pérez, F., \& Fernández, H. (2009). Econometría. Conceptos Básicos (Primera ed.). Medellín: Ecoe. Ediciones.

Palley, Thomas I. (2011) : The contradictions of export-led growth, Public policy brief // Jerome Levy Economics Institute of Bard College, No. 119, ISBN 978-1-936192-17-5

Sant'Anna,. Filomena, \&. Caldeira, Index tracking and enhanced indexing using cointegration and correlation with endogenous portfolio selection, The Quarterly Review of Economics and Finance, Volume 65, 2017, Pages 146-157, ISSN 1062-9769, https://doi.org/10.1016/j. qref.2016.08.008.

Wooldridge, J. (2010). Introducción a la Econometría. Un enfoque moderno. (Cuarta ed.). México: Cengage Learning. 